\title{
ON CLOSING MANIFOLDS FIBERED OVER SURFACES
}

\section{C. THORNTON}

1. Introduction. Given a manifold it is sometimes possible to make identifications on its boundary to obtain a closed manifold. For example any closed $n$-manifold may be obtained from an $n$-cell in this manner [2]. In order to investigate which closed manifolds may be obtained from a class of manifolds with boundary we assume the additional structure of a singular fibering [12]. Analysis of the fundamental group then gives necessary conditions for two closings to be homeomorphic.

2. Singular fiberings. Let $\bar{M}$ be a locally trivial fiber space with fiber $F$, a connected $(n-2)$-manifold, and base $\bar{B}$, a sphere with $g \geqq 0$ handles (or $k>0$ crosscaps) and $r$ boundary components. Further assume $\bar{M}$ can be obtained as follows. Let $B$ be a sphere with $g \geqq 0$ handles ( $k$ crosscaps). Cutting $B$ along a set of loops based at $v_{1}$ gives a $4 g$-gon (2k-gon) $P$ with sides $A_{1} B_{1} A_{1}^{-1} B_{1}^{-1} \cdots B_{g}^{-1}\left(C_{1} C_{1}^{\prime} C_{2} \cdots C_{k} C_{k}^{\prime}\right)$ to be identified in pairs. Removing an open disk $D_{0}$ around $v_{1}$ gives a polygon $\bar{P}$ which is $P$ with a sector of a disk removed from each vertex. $\bar{P} \times F$ is an $n$-manifold on which we shall make some identifications.

Let $\chi: \pi_{1}\left(B, v_{0}\right) \rightarrow$ Automorphisms of $\pi_{1}(F)$ be a homomorphism where $v_{0}$ is the center of $P$ and the automorphisms act on the right of $\pi_{1}(F)$. Let $x, x^{\prime}$ be points on the straight edges of $\bar{P}$ which will be identified in $B$ and let $\alpha$ be the path formed by the straight lines $v_{0}$ to $x$ and $x^{\prime}$ to $v_{0}$ which is a loop in $B$ based at $v_{0}$. Then choose a base point preserving homeomorphism $x \times F \rightarrow x^{\prime} \times F$ which induces $\chi([\alpha]): \pi_{1}(F) \rightarrow \pi_{1}(F)$. Identifying pairs of fibers over the straight edges of $\bar{P}$ by these homeomorphisms gives a manifold $\bar{M}_{0}$ with a boundary $\partial D_{0} \times F$. Now suppose $\partial D_{0} \times F$ is trivially fibered by circles $\omega$ such that $[\omega]=d_{0}+t \in \pi_{1}\left(\partial D_{0} \times F\right)$ where $d_{0}$ generates $\pi_{1}\left(\partial D_{0}\right)$ and $t \in \pi_{1}(F)$. By identifying each $\omega$ on $\partial D_{0} \times F$ to a point we obtain a closed $n$-manifold $M_{0}[1$, p. 229] fibered by $F$ over $B$. The homomorphism $\chi$ is its characteristic and $t \in \pi_{1}(F)$ is the obstruction to a cross section. By removing the fibers over open disks $D_{j}, j=1, \cdots, r$, in $B$ we obtain $\bar{M}$ with $r$ boundary components $\partial D_{j} \times F$.

Suppose $\partial D_{j} \times F$ is trivially fibered by circles $\omega_{j}$ such that $\left[\omega_{j}\right]$ $=n_{j} d_{j}+u_{j} \in \pi_{1}\left(\partial D_{j} \times F\right)$ where $n_{j}$ is a nonzero integer, $d_{j}$ a generator of $\pi_{1}\left(\partial D_{j}\right)$ and $u_{j} \neq 1 \in \pi_{1}(F)$. By identifying each $\omega_{j}$ to a point $\bar{M}$ is

Received by the editors April 14, 1967. 
transformed into another $n$-manifold with one less boundary component. The $\partial D_{j} \times F$ has been replaced by a space $F_{j}$ homeomorphic to $F . F_{j}$ is a singular fiber of type $n_{j}[12]$. Note $F_{j}$ will have a trivial product neighborhood if and only if $n_{j}= \pm 1$. Replacing all boundary components of $\bar{M}$ by singular fibers in this manner we obtain a closed manifold $M$ which we say is singularly fibered by $F$ over a surface.

For example, a locally-trivial fibering of a 4-manifold by a closed surface is fiber preserving homeomorphic to such an $\bar{M}$. If $F$ is taken to be a circle, then the singularly fibered $M$ are the Seifert manifolds [11]. Recently it has been shown by Orlik, Vogt and Zieschang [10] that for Seifert manifolds, with a few exceptions, the fundamental group determines the manifold up to a fiber preserving homeomorphism. In that direction we have the following results for a more general fiber.

\section{Fundamental groups and singular fiberings.}

THEOREM 1. Let $M$ be singularly fibered over a closed surface by a path connected fiber $F$ with $\pi_{1}(F)$ abelian. If the base space is a sphere, torus, or projective plane also assume $r-2>\sum 1 / n_{j}, r \geqq 1$, or $r \geqq 2$ respectively, where $r$ is the number of singular fibers, and the types are $n_{j}, j=1, \cdots, r$. Then $\pi_{1}(M)$ determines the base space, the number of singularities and the types of singularities.

THEOREM 2. Let $M$ be singularly fibered by $F$ over a closed surface $B$. If $\pi_{1}(F)$ can be finitely presented and if $F$ has first Betti number greater than one then $\pi_{1}(M)$ is infinite.

To prove these results we need a presentation of $\pi_{1}(M)$. Suppose $\pi_{1}(F)$ is presented by $\left(s_{l} \mid w_{m}\right), B$ has $g \geqq 0$ handles (or $k>0$ crosscaps), and $M$ has characteristic $\chi: \pi_{1}(B) \rightarrow$ Aut $\pi_{1}(F)$, obstruction term $t \in \pi_{1}(F)$ and singular fibers $F_{j}$ produced by circles $\omega_{j}$ with $\left[\omega_{j}\right]$ $=n_{j} d_{j}+u_{j} \in \pi_{1}\left(\partial D_{j} \times F\right)$. Then a straightforward application of Van Kampen's theorem shows that $\pi_{\mathbf{1}}(M)$ is presented by generators: $a_{i}, b_{i},\left(\right.$ or $\left.c_{i}\right), d_{j}, s_{l} ; i=1, \cdots, g$ (or $\left.k\right), j=1, \cdots, r$, and relations: $\prod\left[a_{i}, b_{i}\right] d_{r}^{-1} \cdots d_{1}^{-1} t$ (or $\left.\prod c_{i}^{2} d_{r}^{-1} \cdots d_{1}^{-1} t\right), w_{m}, d^{n_{i}} u_{j},\left[d_{j}, s_{l}\right]$, and $x^{-1} s_{l} x=s_{l} \chi(x)$ where $x=a_{i}, b_{i}\left(\right.$ or $\left.c_{i}\right)$.

Lemma 1. Let

$$
\begin{aligned}
& K_{1}\left(g ; n_{1}, \cdots, n_{r}\right)=\left(a_{i}, b_{i}, d_{j} \mid \Pi\left[a_{i}, b_{i}\right] d_{1} \cdots d_{r}, d_{j}^{n_{j}}\right) \\
& g>0, r>0 \\
& K_{2}\left(k ; n_{1}, \cdots, n_{r}\right)=\left(c_{i}, d_{j} \mid \prod c_{i}^{2} d_{1} \cdots d_{r}, d_{j}^{n_{j}}\right) \\
& k>0, r>0 \text { and } r \geqq 2 \text { if } k=1 \text {, }
\end{aligned}
$$




$$
\begin{aligned}
K_{3}(g ;-) & =\left(a_{i}, b_{i} \mid \prod\left[a_{i}, b_{i}\right]\right), \quad g \geqq 2, \\
K_{4}(k ;-) & =\left(c_{i} \mid \prod c_{i}^{2}\right), \quad k \geqq 2, \\
K_{5}\left(-; n_{1}, \cdots, n_{r}\right) & =\left(d_{j} \mid d_{1} \cdots d_{r}, d_{j}^{n_{j}}\right), \quad r-2>\sum 1 / n_{j} .
\end{aligned}
$$

Then all of these groups are nonisomorphic in pairs, that is if $K_{i}\left(x ; p_{1} \cdots p_{r}\right) \approx K_{j}\left(y ; q_{1}, \cdots, q_{s}\right)$ then $i=j, x=y, r=s$, and $q_{1}, \cdots, q_{s}$ is a permutation of $p_{1}, \cdots, p_{r}$.

Proof. These follow from known results, e.g. [3, p. 203], [6, p. 239], [8, p. 55], [9, pp. 12-42], and [13].

Lemma 2. A non-Euclidean crystallographic group of the form $K_{i}$ in Lemma 1 contains no nontrivial normal abelian subgroup.

Proof. Let $K$ be of the form $K_{1}, K_{3}$, or $K_{5}$. Then $K$ has a faithful representation as a Fuchsian group of the first kind [6, p. 239], so we may consider it as a group of linear transformations of the upper half plane [7, p. 1]. Therefore any abelian subgroup $A \subset K$ is cyclic [7, p. 15]. Now $K$ has a trivial center since it is the amalgamated free product of two groups, one of which has a trivial center [5, p. 32]. In particular, $A$ is not contained in the center of $K$. Thus there exist elements $g \in K, s \in A$ which do not commute. By [7, p. 9], two elements commute if and only if they have the same fixed points, so $g$ and $s$ have different fixed points. Thus $g s g^{-1}$ has fixed points different than those of $s$ so $g s g^{-1}$ is not in $A$ and $A$ is not normal.

Suppose $K$ is of the form $K_{2}$ or $K_{4}$ and let $A$ be a normal abelian subgroup. $K$ contains a Fuchsian group $F$ as a normal subgroup of index 2. Then $A \cap F$ is a normal abelian subgroup of $F$ and by the argument above $A \cap F=1$. Thus $A$ is presented by $\left(a \mid a^{2}=1\right)$. Since $A$ is normal we have $\mathrm{gag}^{-1}=a$ for all $g \in K$, that is $a$ is in the center of $K$. But if $k \geqq 2, K$ is the free product of $\left(c_{1} \mid-\right)$ and $\left(c_{2}, \cdots, c_{k}, d_{1}, \cdots, d_{r} \mid d_{j_{k}}^{n_{1}}\right)$ (or $\left(c_{2}, \cdots, c_{k} \mid-\right)$ ) amalgamated along subgroups isomorphic to $Z$ generated by $c_{1}^{-2}$ and $c_{2}^{2} \cdots c_{k}^{2} d_{1} \cdots d_{r}$ (or $c_{2}^{2} \cdots c_{k}^{2}$ ). Thus the center of $K$ is torsion free. If $K=\left(c, d_{1}, \cdots\right.$, $\left.d_{r} \mid c^{2} d_{1} \cdots d_{r}, d_{j}^{n_{j}}\right) r \geqq 2$, then $K$ is the free product of $(c \mid-)$ and $\left(d_{1}, \cdots, d_{r} \mid d_{j}^{n_{i}}\right)$ amalgamated along subgroups isomorphic to $Z$ generated by $c^{-2}$ and $d_{1} \cdots d_{r}$. Here $K$ has no center since $\left(d_{1}, \cdots, d_{r} \mid d_{j}^{n_{j}}\right)$ has no center.

Proof of Theorem 1. Let $S$ be the subgroup of $\pi_{1}(M)$ consisting of all words in the $s_{l}$ generators. The presentation of $\pi_{1}(M)$ shows $S$ is a normal abelian subgroup. $S$ is characterized as the unique maximal normal abelian subgroup of $\pi_{1}(M)$. For suppose $T$ is another normal abelian subgroup of $\pi_{1}(M)$ and $S \cap T \neq T$. Then $T S$ is a normal sub- 
group containing $S$ and $T S / S \approx T / S \cap T$ is a nontrivial normal abelian subgroup of $\pi_{1}(M) / S$. But $\pi_{1}(M) / S$ is a group of the type listed in Lemma 2 so it has no nontrivial normal abelian subgroup. Thus $S$ is a characteristic subgroup of $\pi_{1}(M)$ and $\pi_{1}(M) / S$ is independent of the presentation of $\pi_{1}(M)$. By Lemma 1 , the $B, r$, and $n_{1}, \cdots, n_{r}$ are therefore determined.

Proof of Theorem 2. $\pi_{1}(M)$ finite implies $\pi_{1}(B)$ must be finite so either $B=S^{2}$ or $B=P^{2}$. If $B=S^{2}$ then $\pi_{1}(M)=\left(s_{l}, d_{j} \mid d_{j}^{n_{j}} u_{j}, \prod d_{j} t, w_{m}\right.$, $\left.\left[s_{l}, d_{j}\right]\right)$ with $l=1, \cdots, p, m=1, \cdots, q, j=1, \cdots, r$. The Jacobian of $\pi_{1}(M)$ evaluated at the trivializer [3, pp. 198-204] has $p+r$ columns and $r+1+q$ nonzero rows. Since the first Betti number of $F$ is at least 2 , the $g$ rows corresponding to the relations $w_{m}$ have rank at most $p-2$. Thus the Jacobian has rank at most $p+r-1$ and the nullity is at least one. Hence $M$ has Betti number at least 1 and $\pi_{1}(M)$ is infinite.

If $B=P^{2}$ then $\pi_{1}(M)=\left(c, s_{l}, d_{j} \mid c^{2} d_{r}^{-1} \cdots d_{1}^{-1} t, d_{j}^{n_{j}} u_{j}, w_{m},\left[s_{l}, d_{j}\right]\right.$, $\left.c^{-1} s_{l} c=s_{l} \alpha\right)$ where $l=1, \cdots, p, j=1, \cdots, r, m=1, \cdots, q$, $\alpha \in$ Aut $\pi_{1}(F)$ is such that $\alpha^{2}=\mathrm{id}$, and $u_{j}, t \in \pi_{1}(F)$. Adding the relations $s_{l}=1$ we obtain $K=\left(c, d_{j} \mid d_{j}^{n_{j}}, c^{2} d_{r}^{-1} \cdots d_{1}^{-1}\right)$. If $r \geqq 2, K$ is infinite since it is an amalgamated free product. Hence we need only consider $\pi_{1}(M)$ with $B=P^{2}$ and $r=0$ or 1 .

Consider $\pi=\left(c, d, s_{l} \mid c^{2} d^{-1} t, d^{n} u, w_{m},\left[s_{l}, d\right], c^{-1} s_{l} c=s_{l} \alpha\right)$ where $t, u \in \pi_{1}(F), \alpha \in$ Aut $\pi_{1}(F)$ with $\alpha^{2}=$ id. We shall show $\pi$ is a normal extension of $N=\left(s_{l} \mid w_{m},\left[s_{l}, t\right]\right)$ by $\left(c \mid c^{2 n}\right)\left[4\right.$, p. 225]. Since $\alpha^{2}=$ id we can present $\pi$ by $\left(c, s_{l} \mid c^{2 n} t^{n} u, w_{m},\left[s_{l}, t c^{2}\right], c^{-1} s_{l} c=s_{l} \alpha\right)$. Defining $s_{l}^{c}=c^{-1} s_{l} c$ gives an automorphism $\bar{\alpha}$ of $N$ such that $\bar{\alpha}$ fixes $\left(t^{n} u\right)^{-1}$ and its $2 n$th iterate is conjugation by $\left(t^{n} u\right)^{-1}$. Then defining the factor sets $\left(c^{i}, c^{j}\right)=1$ if $i+j \leqq 2 n-1$ and $\left(c^{k}, c^{j}\right)=\left(t^{n} u\right)^{-1}$ if $i+j \geqq 2 n$ specifies a group extension $G$ which is isomorphic to $\pi$ and contains the infinite subgroup $N$. For the case $r=0$ we set $n=1, u=1 \in \pi_{1}(F)$ and omit the relations $\left[s_{l}, d\right]$ and $\left[s_{l}, t\right]$ to obtain $\pi_{1}(F)$ as a subgroup of $\pi$. Note the requirement that first Betti number $\geqq 2$ cannot be dropped. Seifert shows with $F$ a circle we can singularly fiber a three sphere [11, p. 206].

\section{REFERENCES}

1. A. Aeppli, Modifikation von reelen und komplexen Mannigfaltigkeiten, Comment. Math. Helv. 31 (1957), 219-301.

2. M. Brown, "A mapping theorem for untriangulated manifolds," pp. 92-94, in Topology of 3-manifolds and related topics, Prentice-Hall, Englewood Cliffs, N. J., 1962.

3. R. H. Fox, Free differential calculus. II, Ann. of Math. 59 (1954), 196-210.

4. M. Hall, The theory of groups, Macmillan, New York, 1959. 
5. A. G. Kurosh, The theory of groups, Vol. 2, Chelsea, New York, 1956.

6. J. Lehner, Discontinuous groups and automorphic functions, Math. Surveys No. 8, Amer. Math. Soc., Providence, R. I., 1964.

7. - A short course in automorphic functions, Holt, New York, 1966.

8. A. M. Macbeath, Discontinuous groups and birational transformations, Mimeographed Notes, Dundee, 1961.

9. P. P. Orlik, Necessary conditions for the homeomorphism of Seifert-manifolds, Thesis, Univ. of Michigan, Ann Arbor, 1966.

10. P. P. Orlik, E. Vogt and H. Zieschang, Zur Topologie Gefaserter Dreidimensionaler Mannigfaltigkeiten, Topology 6 (1967), 49-65.

11. H. Seifert, Topologie Dreidimensionaler Gefaster Räume, Acta Math. 60(1933), 147-238.

12. M. C. Thornton, Singularly fibered manifolds, Illinois J. Math. 11 (1967), 189201.

13. H. Zieschang, Eben diskontinuierliche Gruppen und ebene Gruppenbilder, Uspehi Mat. Nauk 21 (1966), 195-212.

UNIVERSITY OF WISCONSIN 